\title{
Similarity Registration for Shapes Based on Signed Distance Functions
}

\author{
Sasan Mahmoodi ${ }^{1}$, Muayed S. Al-Huseiny ${ }^{2}$, and Mark S. Nixon ${ }^{1}$ \\ ${ }^{1}$ School of Electronic and Computer Science, University of Southampton, UK \\ ${ }^{2}$ Computer and Software Engineering Department, University of Mustansiriyah, Iraq
}

\begin{abstract}
A fast algorithm for similarity registration for shapes with various topologies is put forward in this paper. Fourier transform and Geometric moments are explored here to calculate the rotation, scaling and translation parameters to register two shapes by minimizing a dissimilarity measure introduced in the literature. Shapes are represented by signed distance functions. In comparison with the algorithms in the literature, the algorithm proposed here demonstrates superior performance for the registration of two shapes with various topologies as well as two shapes, each containing various and different numbers of shape components. The registration process using this algorithm is robust in comparison with the shape registration algorithms in the literature and is as fast as a couple of FFTs.
\end{abstract}

\section{Introduction}

Shape registration may be regarded as the result of a point-wise transformation between a reference and an observed shape [2]-[3]. Registration algorithms established on matching contour points (contour-based) are cited in the literature due to their fast convergence (e.g. see [5], [6], and [7]). The very fact though that such algorithms rely on contour points only (require point correspondence) makes these methods vulnerable to topological changes in shapes. More recent work tends towards using SDF (SDF-based) (e.g. see [1], [4], [8] and [9]). These SDF-based algorithms usually minimize the distance between the SDFs iteratively for instance by using a gradient descent algorithm. The SDF-based shape registration methods (for example the seminal work in [1]) are in general capable of dealing with shapes that have various Euler characteristic numbers (various topologies). However, as the complexity of shapes increases, the possibility of convergence to local minima becomes more likely. These methods are somewhat slow and sometimes lack stability due to their iterative nature. Also, the implementation is slightly difficult to manage because there is no unique method for a universal stopping criterion that is applicable for every case (see [4] for more details). The algorithm proposed in this paper however is fast, reliable, robust to local minima problem and can perform registration successfully between shapes with various topologies. The paper is organized as follows: The registration problem is stated in section 2. The proposed algorithm is presented in section 3. Important considerations noted for implementation are discussed in section 4 . The algorithm is evaluated in section 5, and finally the paper concludes in section 6 . 


\section{The Statement of the Problem}

The signed distance function (SDF) of a shape $p$ is defined as:

$$
\phi_{p}(x, y)=\left\{\begin{array}{cl}
D_{E}((x, y), B), & (x, y) \in I_{p}, \\
-D_{E}((x, y), B), & (x, y) \in \Omega-I_{p},
\end{array}\right\}
$$

where $\Omega$ is the bounded domain, $D_{E}$ stands for the minimum Euclidean distance between the perimeter $B$ of the shape $p$ and any point in domain $\Omega$, and $I_{p}$ is the subset of $\Omega$ representing the interior of the shape [1]. Registration between two shapes aims to retrieve transform parameters $s, \theta, T_{x}$ and $T_{y}$ (scaling, rotation, and translations along $x$ and $y$ axes respectively) minimizing a dissimilarity measure between $\phi_{p}$ and $\phi_{q}$ introduced in [1] and given in (2),

$$
E=\iint_{\Omega}\left|\phi_{p}(x, y)-\frac{1}{s} \phi_{q}\left(s R_{\theta}\left(x+T_{x}, y+T_{y}\right)\right)\right|^{2} d x d y
$$

such that,

$$
\left(\hat{\theta}, \hat{s}, \hat{T}_{x}, \hat{T}_{y}\right)=\underset{\theta, s T_{x}, T_{y}}{\arg \min } E
$$

where $\Omega, \hat{\theta}, \hat{s}, \hat{T}_{x}, \hat{T}_{y}$ and $R_{\theta}$ are image domain, the estimated angle, scale, translation parameters, and a conventional rotation (transform) matrix respectively,

$$
R_{\theta}=\left[\begin{array}{cc}
\cos \theta & -\sin \theta \\
\sin \theta & \cos \theta
\end{array}\right] .
$$

\section{Registration Method}

The minimization of dissimilarity measure (2) with respect to the desired parameters can be directly and iteratively implemented as demonstrated in [1]. Such an implementation is slow, unreliable (may fall into local minima), and difficult to tune [4][11]. The algorithm presented here on the other hand, suggests linear methods to estimate the registration parameters minimizing the dissimilarity measure in (2).

\subsection{Rotation}

For simplicity, let the objective function in (2) be a function of only $\theta$ :

$$
E_{\theta}=\iint_{\Omega}\left|\phi_{p}(x, y)-\phi_{q}\left(R_{\theta}(x, y)\right)\right|^{2} d x d y
$$

Rotation in Cartesian coordinates is equivalent to displacement of the angular component in polar coordinates [10]. 
Shapes $p$ and $q$ are initially centered at the origin of the coordinate system. Centralized shapes are then mapped to polar coordinates i.e., $\hat{\phi}_{p}(\rho, \omega)$ and $\hat{\phi}_{q}(\rho, \omega)$ such that $x=\rho \cos \omega$ and $y=\rho \sin \omega$. In theorem 1, we prove that the rotation angle minimizing term (5) minimizes term (4):

$$
E_{\theta}=\int_{0}^{+\infty} \int_{0}^{2 \pi}\left|\hat{\phi}_{p}(\rho, \omega)-\hat{\phi}_{q}((\rho, \omega+\theta))\right|^{2} \mathrm{~d} \rho \mathrm{d} \omega,
$$

Theorem 1: The rotation angle minimizing term (5) is the minimizer of term (4) where $\Omega=R^{2}$.

The proof is presented in the appendix. We use the result obtained from theorem 1 to propose an algorithm to estimate the desired rotation angle $\theta$. To this end, let us denote $\bar{\phi}_{p}$ a normalized instance of $\hat{\phi}_{p}$ :

$$
\bar{\phi}_{p}(\rho, \omega)=\frac{\hat{\phi}_{p}(\rho, \omega)}{\sqrt{\int_{\rho} \int_{\omega}\left|\hat{\phi}_{p}(\rho, \omega)\right|^{2} \mathrm{~d} \rho \mathrm{d} \omega}} .
$$

Also, let $\beta$ be the scale factor ( $\beta$ is a function of the desirable rotation angle $(\theta)$ between $\bar{\phi}_{p}$ and $\hat{\phi}_{\mathrm{q}}$, i.e., $\beta(\theta)=\left\langle\hat{\phi}_{q}, \bar{\phi}_{p}\right\rangle=\int_{\rho} \int_{\omega}\left(\hat{\phi}_{q}(\rho, \omega) \bar{\phi}_{p}(\rho, \omega+\theta)\right) \mathrm{d} \rho \mathrm{d} \omega$.

The desirable rotation angle is estimated by minimizing the dissimilarity term $E_{\theta}$ between $\hat{\phi}_{p}$ and $\hat{\phi}_{\mathrm{q}}$ in (5):

$$
\begin{aligned}
E_{\theta} & =\int_{\rho} \int_{\omega}\left|\hat{\phi}_{q}-\bar{\phi}_{p}\right|^{2} \mathrm{~d} \rho \mathrm{d} \omega, \\
& =\int_{\rho} \int_{\omega}\left(\left|\hat{\phi}_{q}\right|^{2}-2 \hat{\phi_{q}} \bar{\phi}_{p}+\left|\bar{\phi}_{p}\right|^{2}\right) \mathrm{d} \rho \mathrm{d} \omega .
\end{aligned}
$$

From (6), $\iint_{\rho}\left|\bar{\phi}_{p}\right|^{2} d \rho d \omega=1$; also, as defined above, $\left\langle\hat{\phi}_{q}, \bar{\phi}_{p}\right\rangle=\beta$, therefore:

$$
E_{\theta}=\int_{\rho} \int_{\omega}\left|\hat{\phi}_{q}\right|^{2} \mathrm{~d} \rho \mathrm{d} \omega-2 \beta(\theta) .
$$

Since the first two terms in (8) are independent of $\theta$, the minimization of $E_{\theta}$ is equivalent to the maximization of $\beta$, i.e., the optimal rotation $\hat{\theta}$ is estimated by maximizing $\beta$.

The Fourier transform is employed here to compute the local maxima of $\beta$. Let the Fourier transform of $\hat{\phi}_{q}$ and $\bar{\phi}_{p}$ be $\hat{\psi}_{q}\left(\xi_{1}, \xi_{2}\right)$ and $\bar{\psi}_{p}\left(\xi_{1}, \xi_{2}\right)$ respectively, such that,

$$
\begin{gathered}
\hat{\psi}_{q}\left(\xi_{1}, \xi_{2}\right)=\int_{\rho} \int_{\omega} \hat{\phi}_{q}(\rho, \omega) e^{-i\left(\rho \xi_{1}+\omega \xi_{2}\right) 2 \pi} \mathrm{d} \rho \mathrm{d} \omega . \\
\bar{\psi}_{p}\left(\xi_{1}, \xi_{2}\right)=\int_{\rho} \int_{\omega} \bar{\phi}_{p}(\rho, \omega) e^{-i\left(\rho \xi_{1}+\omega \xi_{2}\right) 2 \pi} \mathrm{d} \rho \mathrm{d} \omega,
\end{gathered}
$$


According to Parseval's theorem, expression (11) holds,

$$
\begin{aligned}
\beta(\theta) & =\int_{\rho} \int_{\omega}\left(\hat{\phi}_{q}(\rho, \omega) \bar{\phi}_{p}(\rho, \omega+\theta)\right) \mathrm{d} \rho \mathrm{d} \omega \\
& =\int_{\xi_{1}} \int_{\xi_{2}}\left(\hat{\psi}_{q}\left(\xi_{1}, \xi_{2}\right) \bar{\psi}_{p}^{*}\left(\xi_{1}, \xi_{2}\right) e^{2 \pi i \zeta_{2} \theta}\right) \mathrm{d} \xi_{1} \mathrm{~d} \xi_{2},
\end{aligned}
$$

where $(*)$ denotes the complex conjugate.

Hence, from (11), $\hat{\theta}$ is estimated as in (12),

$$
\hat{\theta}=\underset{\theta}{\operatorname{argmax}} \beta=\underset{\theta}{\operatorname{argmax}} \int_{\xi_{1}} \int_{\xi_{2}}\left(\hat{\psi}_{q} \bar{\psi}_{p}^{*} e^{i \theta}\right) \mathrm{d} \xi_{1} \mathrm{~d} \xi_{2} .
$$

\subsection{Scale}

Dissimilarity measure (2) is minimized with respect to $s$ to compute the relative scale between the two given shapes. This measure is expressed in (13) in terms of s for the sake of simplicity:

$$
E_{s}=\iint\left|\phi_{p}(x, y)-\frac{1}{s} \phi_{q}(s(x, y))\right|^{2} d x d y,
$$

where the relation between two shapes' SDFs which have different scales is known to be:

$$
s \hat{\phi}_{p}(x, y)=\hat{\phi}_{q}(s x, s y),
$$

where $\hat{\phi}_{p}$ and $\hat{\phi}_{q}$ are the centralized versions of $\phi_{p}$ and $\phi_{q}$. In theorem 2, we prove that the scaling parameter $s$ minimizing the following term is a minimizer of term (13):

$$
E_{s}^{\prime}=\left|\sum_{m=0}^{M} \sum_{n=0}^{N}\left(M_{m n}^{p}-\frac{M_{m n}^{q}}{s^{m+n+3}}\right)\right|^{2}
$$

where $M_{m n}^{p}$ and $M_{m n}^{q}$ are respectively the $(m+n)^{\text {th }}$ order geometrical moments of $\hat{\phi}_{p}(x, y)$ and $\hat{\phi}_{q}(x, y)$ defined as:

$$
\begin{aligned}
M_{m n}^{p} & =\iint_{\Lambda} x^{m} y^{n} \hat{\phi}_{p}(x, y) d x d y \\
M_{m n}^{q} & =\iint_{\Lambda} x^{m} y^{n} \hat{\phi}_{q}(x, y) d x d y
\end{aligned}
$$

Theorem 2: The scaling parameter minimizing term (13) is also the minimizer of term (15). 
The proof is presented in the appendix. This distance term given in (15) is not linear with respect to $s$. By using a change of variable, (15) is linearized with respect to $\hat{s}=\log s$,

$$
\hat{E}_{s}=\left|\sum_{m=0}^{M} \sum_{n=0}^{N} \log \left(\frac{M_{m, n}^{q}}{M_{m, n}^{p}}\right)-\hat{s}(m+n+3)\right|^{2},
$$

Therefore, $s$ minimizing $E_{s}^{\prime}$, is computed as:

$$
s=\exp \left(\frac{\sum_{m} \sum_{n} \log \left(\frac{M_{m n}^{q}}{M_{m n}^{p}}\right)}{\sum_{m} \sum_{n}(m+n+3)}\right) .
$$

\subsection{Translation}

The scale and rotation information computed previously in sections 3.1 and 3.2 are used to fix the scaling and rotation discrepancies between shapes. For translation parameters only, term (2) thus reduces to Eq.(18),

$$
E_{T_{x}, T_{y}}=\iint\left|\phi_{p}(x, y)-\phi_{q}\left(\left(x+T_{x}, y+T_{y}\right)\right)\right|^{2} \mathrm{~d} x \mathrm{~d} y,
$$

A similar approach to that employed in 3.1 is used here to estimate translation parameters. Let $\bar{\phi}_{p}$ denote a normalized version of $\phi_{p}$, that is:

$$
\bar{\phi}_{p}(x, y)=\frac{\phi_{p}(x, y)}{\sqrt{\int_{x, y}\left|\phi_{p}(x, y)\right|^{2} \mathrm{~d} x d y}} .
$$

By using a similar argument as the one employed in section 3.1, the translation parameters are calculated as:

$$
\left.\mid \begin{array}{ll}
\hat{T}_{x} & \hat{T}_{y}
\end{array}\right]=\underset{T_{x}, T_{y}}{\operatorname{argmax}} \int_{\lambda_{x}} \int_{\lambda_{y}}\left(\psi_{q}\left(\lambda_{x}, \lambda_{y}\right) \bar{\psi}_{p}^{*}\left(\lambda_{x}, \lambda_{y}\right) e^{2 \pi i\left(T_{x} \lambda_{x}+T_{y} \lambda_{y}\right)}\right) \mathrm{d} \lambda_{x} \mathrm{~d} \lambda_{y},
$$

where $\hat{T}_{x}, \hat{T}_{y} \psi_{q}\left(\lambda_{x}, \lambda_{y}\right), \bar{\psi}_{p}\left(\lambda_{x}, \lambda_{y}\right), \lambda_{x}, \lambda_{y}$ and $*$ represent the estimated optimal translation parameters, 2D Fourier transform of $\phi_{q}$ and $\bar{\phi}_{p}$, spatial frequencies and complex conjugate respectively.

\section{$4 \quad$ Implementation Issues}

$\mathrm{W}$ notice that the theorems proved in this paper, indicate that the transformation parameters can be calculated by using a linear method. It is therefore important to note 
that this linearity is not an assumption and therefore it is applicable to general cases. The employment of continuous Fourier transform in the computation of the rotation and translation is not numerically tractable. Fast Fourier transform (FFT) is employed instead in this paper. Accordingly, the definition of SDFs is modified to accommodate the periodicity property associated with FFT: Let $\Omega$ be the shape domain. This domain is partitioned by the shape perimeter into two regions, the shape interior $I$ and the background, and let $\phi: \Omega \rightarrow \Re^{+}$be a Lipschitz function as defined in (21):

$$
\phi(x, y)=\left\{\begin{array}{cc}
D_{E}((x, y), B), & (x, y) \in I, \\
0, & (x, y) \in \Omega-I,
\end{array}\right\}
$$

where $D_{E}$, as mentioned earlier, stands for the minimum Euclidean distance between shape perimeter $B$ and any point inside the shape.

Registration parameters are computed in the following order: rotation, scaling and finally translation. Observed shapes are fixed accordingly after computing each parameter. In the case of scale it is important to remove the variance in shapes due to differences in translation (by centralizing the shapes) and rotation since these variances affect the computed moments. A Matlab ${ }^{\circledR}$ (version 7) implementation code of the algorithm proposed in this paper is available: at http://users.ecs.soton.ac.uk/sm3/SDFShapeRegistration.zip. The practical limitation of this approach is that it may fall into local minima, because dissimilarity measure (2) is also associated with the local minima. The last but not the least issue is that the order in which the registration parameters are calculated does not change the final result.

\section{$5 \quad$ Results}

The proposed algorithm is evaluated by using a set of problems to address issues common to shapes. In the subsequent examples the moments up to the tenth order (up to $m=n=5$ ) are used to compute the scale parameter $s$ presented in section 3.2. Indeed, the higher the number of moments is, the more accurately $s$ is estimated and the more expensive the algorithm becomes.

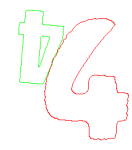

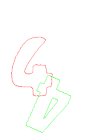

(b)

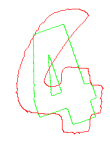

(c)

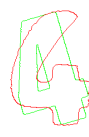

(d)

Fig. 1. Registration of shapes with different topologies (size $=300$ X 300). (a) initial shapes, (b) registration by using the contour-based method in [6], (c) registration by using the SDF-based method in [1] (d) registration by using the approach proposed here. 


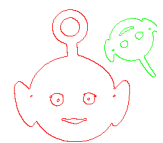

(a)

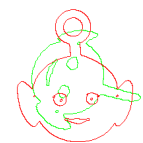

(b)

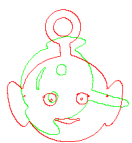

(c)

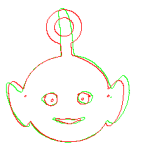

(d)

Fig. 2. Registration of shapes with different topologies ( (b) registration by using the contour-based method in [6], (c) registration by using the SDFbased method in [1] (d) registration by using the approach proposed here.

For the sake of comparison, the results of the proposed technique are compared to those utilized by two well-established shape registration algorithms. The first uses contours for representing shapes, (e.g. see [6]) referred here as the contour-based method. The other algorithm used here for comparison is based on SDFs (see [1] for more details) and referred here as the SDF-based method. For better visualization, the boundaries of the shapes rather than the SDFs are used in all figures in this paper. Contours of the reference shapes are presented in red, and those of the observed shapes are shown in green. The first experiment investigates the impact of topological difference on the registration outcomes. In Figure (1-a), the reference shape is an open number 4 with Euler number unity. The observed shape is a closed number 4 with Euler number zero. The registration by using the contour-based method in [6] shown in Figure (1-b) is not satisfactory; the two shapes are completely misaligned here. A similar example is shown in Figure (2-a), the shapes of the two characters have different topologies. In this example, we refer to the results of applying the SDF-based registration algorithm in [1] to this problem. Figure (2-c) shows a typical case of the local minima issue usually attributed to the iterative procedure of the work presented in [1]. Figures (1-d) and (2-d) on the other hand demonstrate that in both cases the shapes are aligned optimally by using the approach proposed here. This robustness can be due to the fact that the algorithm proposed here employs regions (SDF) rather than boundaries to find the optimal values for registration.

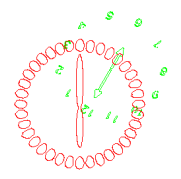

(a)

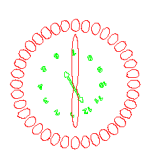

(b)

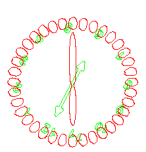

(c)

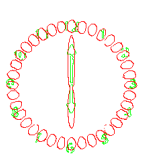

(d)

Fig. 3. Registration of clocks with different number of components (size $=300 \mathrm{X} 300$ ), (a) an initial state, and (b) registration of (a) by using contour-based technique in [6], (c) registration of (a) by using the SDF-based algorithm in [1], and (d) registration of (a) by using the algorithm proposed here 
The next experiment concerns the registration of two complex shapes containing multiple simple shapes (components). The contour-based method in [6] requires point correspondence of the contours. Since the number of components in the two shapes in question (see Figure (3-a)) is different for each shape, the parts with no corresponding counterparts remain untouched. For the sake of comparison, these shapes are treated here as a single entity and registered directly by using the method proposed by the authors in [6]. Figure (3-b) depicts the meaningless alignment by using the contourbased algorithm caused primarily by the lack of contour points' correspondence. The algorithm in [1] on the other hand due to the use of SDFs is capable of handling complex shapes. However, the increased complexity increases the tendency towards stopping at local minima (see figure (3-c)). The results shown by the example in Figure (4) are also consistent with the previous analysis. In either case the algorithm proposed here possesses the capacity to register the shapes quickly and accurately as depicted in Figures (3-d), and (4-d). Our algorithm proposed here is as fast as a couple of FFT operations; however the iterative SDF based algorithm may take a long time to converge, depending on the complexity and size of the shapes.

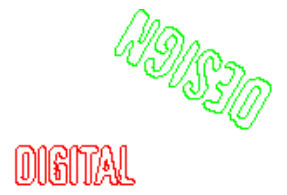

(a)

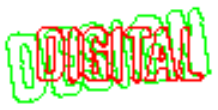

(c)

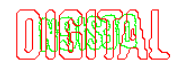

(b)

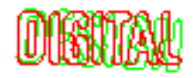

(d)

Fig. 4. Registration of words with various number of letters (size $=300 \times 300$ ), (a) original shapes (b) registration by using contour-based algorithm in [6], (c) registration by using SDFbased algorithm in [1]. (d) registration by using algorithm presented here.

\section{Conclusion}

This paper presents a fast reliable shape registration algorithm. It employs implicit representation of shapes by using signed distance functions (SDFs). The desirable parameters are calculated by minimizing a dissimilarity measure between two shapes. To achieve that, linear orthogonal transformations are employed to minimize the dissimilarity measures. This technique is based on spectral phase correlation and geometric moments to compute registration parameters individually. A modified signed distance function definition is adopted here to satisfy the requirements of the optimization strategy used in this context. The proposed approach is tested satisfactorily on problems such as complex shapes and shapes with various topologies. These shapes 
prove difficult to register by using contour based methods. This work is also examined in comparison with a SDF based iterative registration algorithm in the literature. The evaluation data demonstrate the reliability, stability and speed of convergence of the proposed registration algorithm. The registration technique presented in this paper is as fast as a couple of FFTs. For the future work, this algorithm can be developed to account for 3D shape registration problems

\section{$7 \quad$ Appendix}

\section{Proof of Theorem 1:}

Dissimilarity measure (4) can be written in polar coordinate system, i.e.:

$$
E_{\theta}=\iint_{R^{2}}\left|\phi_{p}(x, y)-\phi_{q}\left(R_{\theta}(x, y)\right)\right|^{2} d x d y=\int_{0}^{+\infty} \int_{0}^{2 \pi}\left|\phi_{p}(\rho, \omega)-\phi_{q}\left(R_{\theta}(\rho, \omega)\right)\right|^{2} \rho d \rho d \omega
$$

where $\rho$ and $\omega$ are polar coordinates so that $x=\rho \cos \omega$, and $y=\rho \sin \omega$.

In a polar coordinate system, term (A-1) can be written as:

$$
E_{\theta}=\operatorname{Lim}_{L \rightarrow+\infty} \int_{0}^{L} \int_{0}^{2 \pi}\left|\phi_{p}(\rho, \omega)-\phi_{q}(\rho, \theta+\omega)\right|^{2} \rho d \rho d \omega<\operatorname{Lim}_{L \rightarrow+\infty} L \int_{0}^{L} \int_{0}^{2 \pi}\left|\phi_{p}(\rho, \omega)-\phi_{q}(\rho, \theta+\omega)\right|^{2} d \rho d \omega
$$

It is easy to see from (A-1) and (A-2) that a parameter $\hat{\theta}$ minimizing

$$
\begin{aligned}
& \int_{0}^{+\infty} \int_{0}^{2 \pi}\left|\phi_{p}(\rho, \omega)-\phi_{q}(\rho, \theta+\omega)\right|^{2} d \rho d \omega \quad \text { is } \quad \text { a minimizer of the term } \\
& \iint_{R^{2}}\left|\phi_{p}(x, y)-\phi_{q}\left(R_{\theta}(x, y)\right)\right|^{2} d x d y
\end{aligned}
$$

\section{Proof of Theorem 2:}

Before proving theorem 2, we need to visit theorem 3:

Theorem 3: Let a geometrical moment with orders $m$ and $n$ of signed distance function $(S D F) \phi_{q}(x, y): \Omega \rightarrow R$ be $M_{m n}^{q}$. The geometrical moment with orders $m$ and $n$ of the scaled SDF with scaling parameter $s$ is $\frac{M_{m n}^{q}}{s^{m+n+3}}$.

\section{Proof:}

$$
M_{m n}^{q}=\iint_{\Omega} x^{m} y^{n} \phi_{q}(x, y) d x d y
$$

The $m^{\text {th }}$ and $n^{\text {th }}$ order moment of the scaled SDF $\frac{1}{s} \phi_{q}(s x, s y)$ is therefore calculated as:

$$
M_{m n}^{\prime q}=\iint_{\Omega} x^{m} y^{n}\left(\frac{1}{s} \phi_{q}(s x, s y)\right) d x d y
$$


By changing the variables $X=s x$ and $Y=s y$, equation (A-4) is rewritten as:

$$
M_{m n}^{\prime q}=\iint_{\Omega}\left(\frac{X}{s}\right)^{m}\left(\frac{Y}{s}\right)^{n}\left(\frac{1}{s} \phi_{q}(X, Y)\right) \frac{d X d Y}{s^{2}}=\frac{1}{s^{m+n+3}} \iint_{\Omega} X^{m} Y^{n} \phi_{q}(X, Y) d X d Y=\frac{M_{m n}^{q}}{s^{m+n+3}}
$$

Proof of theorem 2: The signed distance functions $\phi_{p}(x, y)$ and $\phi_{q}(x, y)$ can be approximated in terms of their geometrical moments, i.e.:

$$
\phi_{i}(x, y) \approx \sum_{m=0}^{M} \sum_{n=0}^{N} M_{m n}^{i} x^{m} y^{n}
$$

where $M_{m n}^{i}=\iint_{\Omega} x^{m} y^{n} \phi_{i}(x, y) d x d y$ and $i$ can be $p$ or $q . M$ and $N$ are the total number of geometrical moments around the axes $x$ and $y$. If equations (14) are substituted in equation (13), by using the result of theorem 3, one can obtain:

$$
E_{s} \approx \iint_{\Omega}\left|\sum_{m=0}^{M} \sum_{n=0}^{N} M_{m n}^{p} x^{m} y^{n}-\sum_{m=0}^{M} \sum_{n=0}^{N} \frac{M_{m n}^{q}}{s^{m+n+3}} x^{m} y^{n}\right|^{2} d x d y=\iiint_{\Omega}\left|\sum_{m=0}^{M} \sum_{n=0}^{N}\left(M_{m n}^{p}-\frac{M_{m n}^{q}}{s^{m+n+3}}\right) x^{m} y^{n}\right|^{2} d x d y
$$

By letting $a_{m n}$ denote $\left(M_{m n}^{p}-\frac{M_{m n}^{q}}{s^{m+n+3}}\right)$, equation (A-7) can be written as:

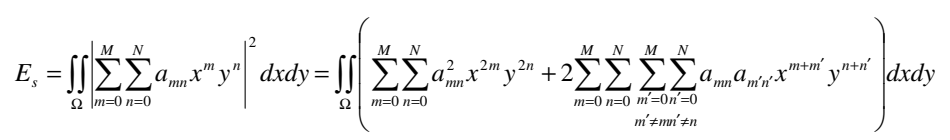

Without loss of generality, for $\Omega=[0, L] \times[0, H], E_{s}$ in $(\mathrm{A}-8)$ can be calculated as:

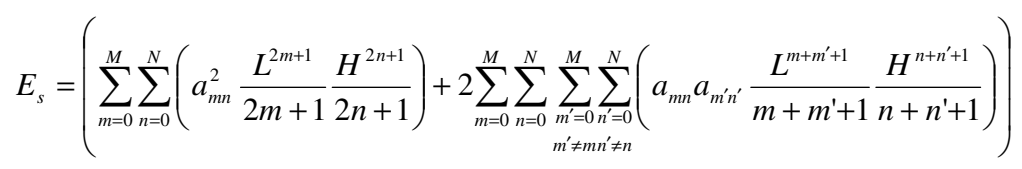

It is easy to conclude from (A-9) that

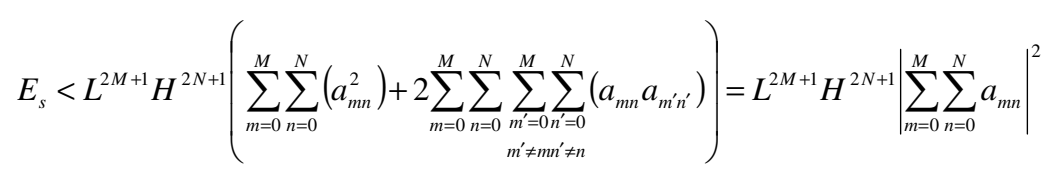

By recalling that the term $a_{m n}=\left(M_{m n}^{p}-\frac{M_{m n}^{q}}{s^{m+n+3}}\right)$ is the only term which is a function of the scaling parameter $s$ and from inequality (A-10), it is straightforward to see that the scaling parameter $s$ minimizing $\left|\sum_{m=0}^{M} \sum_{n=0}^{N}\left(M_{m n}^{p}-\frac{M_{m n}^{q}}{s^{m+n+3}}\right)\right|^{2}$ is a minimizer of $E_{s}$ given in equation (13). 


\section{References}

1. Paragios, N., Rousson, M., Ramesh, V.: Non-rigid registration using distance functions. Computer Vision and Image Understanding 89(2-3), 142-165 (2003)

2. Brown, L.G.: A survey of image registration techniques. ACM Comput. Surv. 24(4), 325376 (1992)

3. Zitova, B.: Image registration methods: a survey. Image and Vision Computing 21(11), 977-1000 (2003)

4. Cremers, D., Osher, S., Soatto, S.: Kernel Density Estimation and Intrinsic Alignment for Shape Priors in Level Set Segmentation. International Journal of Computer Vision 69(3), 335-351 (2006)

5. Marques, J.S., Abrantes, A.J.: Shape alignment - Optimal initial point and pose estimation. Pattern Recognition Letters 18(1), 49-53 (1997)

6. Markovsky, I., Mahmoodi, S.: Least-Squares Contour Alignment. IEEE Signal Processing Letters 16(1), 41-44 (2009)

7. Hui, L., Manjunath, B.S., Mitra, S.K.: A contour-based approach to multisensor image registration. IEEE Transactions on Image Processing 4(3), 320-334 (1995)

8. Vemuri, B.C.: Image registration via level-set motion: applications to atlas-based segmentation. Medical Image Analysis 7(1), 1-20 (2003)

9. El Munim, H.A., Farag, A.A.: Shape Representation and Registration using Vector Distance Functions. In: IEEE Conference in Pattern Recognition (2007)

10. Casasent, D., Psaltis, D.: Position, rotation, and scale invariant optical correlation. Applied Optics 15(7), 1795-1799 (1976)

11. Al-Huseiny, M.S., Mahmoodi, S., Nixon, M.S.: Robust Rigid Shape Registration Method Using a Level Set Formulation. In: Bebis, G., Boyle, R., Parvin, B., Koracin, D., Chung, R., Hammound, R., Hussain, M., Kar-Han, T., Crawfis, R., Thalmann, D., Kao, D., Avila, L. (eds.) ISVC 2010. LNCS, vol. 6454, pp. 252-261. Springer, Heidelberg (2010) 